\title{
Cuasimercados en el ámbito educativo: una aproximación a la experiencia británica
}

\section{Javier Salinas Jiménez * y Carmen Pérez Esparrells **}

\section{Introducción}

El crecimiento de los programas de gasto público que ha tenido lugar en los países de la OCDE en las últimas décadas ha originado un intenso debate, tanto en foros académicos como de las propias Administraciones públicas, acerca del nivel de eficiencia con el que se administran los recursos públicos. Como consecuencia de ello, se han producido una serie de reformas en profundidad de la gestión de las Administraciones públicas con la finalidad de introducir mayor racionalidad económica en su funcionamiento. Entre estas reformas destaca la introducción en varios países de la OCDE de mecanismos de simulación de mercados en el ámbito público, con el objeto de lograr incrementos en la eficiencia y productividad en el Sector Público. Estos mecanismos de cuasimercados o mercados internos han sido aplicados con profusión en sectores tan diversos como la educación, la sanidad o los servicios sociales.

Los mecanismos de cuasimercado no son algo homogéneo. Con carácter general, tal y como señalan ALBI et al. (1997), los cuasimercados pueden ser definidos como aquellos instrumentos de gestión, o diseños organizativos públicos, en los que está presente al menos una característica significativa de los mercados como pueden ser la competencia, el uso de señales de precio, la toma descentralizada de decisiones o los incentivos monetarios.

BARTLETT (1993) indica que quizás la mejor forma de definir un mercado interno sea describir sus características fundamentales, entre las que se encuentran:

- Separación entre la financiación/provisión y producción o suministro de los servicios públicos;
- una vez determinado en el presupuesto el volumen de recursos de que se dispone para una actividad determinada, los recursos no se asignan a los proveedores mediante una decisión burocrática, sino por la elección de los usuarios. Así, en el caso de la educación, los recursos deberían seguir a los alumnos;

- los recursos son asignados por actividad y mercado. De esta forma, por ejemplo, los vales o bonos escolares no pueden ser transformados en dinero y no tienen valor alguno fuera del sistema educativo;

- los precios en el mercado interno no se determinan generalmente en función de la oferta y la demanda, sino que son negociados entre el proveedor de los fondos y el comprador en el marco de una estructura fuertemente regulada;

- los mercados son «internos», en el sentido de que las transacciones realizadas en ellos permanecen dentro del ámbito público.

El objetivo último de los mercados internos es lograr, mediante los estímulos de la competencia, niveles mayores de eficacia, eficiencia y respuesta a las necesidades de los ciudadanos que los obtenidos a través del modelo burocrático tradicional. Como nos recuerdan SAPPIGTON y STiglitz (1987), estos objetivos podrán ser alcanzados en función de que se cumplan ciertos requisitos previos, entre los que destacan la existencia de un nivel suficiente de competencia entre los suministradores de los servicios; la cuantía de los costes de transacción asociados a los contratos así como los derivados de administrar, regular, supervisar y controlar el cuasimercado (que deben ser inferiores a las ganancias de eficiencia que implique la propia introducción del cuasimercado); y la estructura administrativa, política y legal que debería ofrecer protección a los 
consumidores y motivar a los agentes del mercado para hacer que el sistema funcione de forma eficiente mediante la actuación de la competencia. Además, como apuntan BARLETT y LE GRAND (1993), en los mercados internos relacionados con la provisión de servicios sociales, la introducción de los mecanismos de mercado no debería originar desigualdades mayores en el acceso a dichos servicios que las que se producen con el sistema de provisión pública directa.

En esta línea, el objetivo del presente trabajo es analizar los efectos de los mecanismos de cuasimercado en el ámbito de la educación. Nuestro análisis se limitará a estudiar los aspectos normativos de los cuasimercados, en general, y aplicados a la educación, en particular, así como a las cuestiones positivas relativas a la experiencia educativa británica puesta en marcha a finales de los años ochenta. Las principales reformas se introdujeron a través de la Education Reform Act (1988), que pretendía aumentar la autonomía de los centros educativos y la capacidad de elección de los padres, y se extendieron, a partir de 1992, con la publicación del Libro Blanco titulado Choice and Diversity. El examen del estado de la cuestión que se presenta parte de un análisis descriptivo del sector educativo antes y después de la Reforma. A partir del mismo, se analizan los principales efectos producidos por la introducción de los mecanismos de cuasimercado. De esta forma, se examinan los principales efectos de la reforma sobre la eficiencia y la productividad, razones fundamentales para la introducción de estos mecanismos, sin olvidar tampoco aquellos efectos que puedan producirse sobre la equidad.

\section{Aproximación a los cuasimercados. Aplicación en el ámbito de la educación}

Numerosos son los autores que han definido este reciente concepto. En esencia, todos ellos coinciden en identificar cuasimercado con una «emulación» por parte del Sector Público de los mecanismos de mercado, en los entornos que generan fallos insuperables en el terreno de la eficiencia y la equidad por sus características particulares. En el caso de la educación su objetivo principal es una mejora de los niveles de eficiencia y productividad en los centros asegurándose que dichos incrementos no se produzcan a expensas de los niveles de equidad.

La eficiencia perseguida por los cuasimercados o mercados internos puede ser de dos clases. Por una parte, se pretende una optimización de la producción, logrando una eficiencia productiva a través de una reducción de costes, o de un incremento en la calidad o cantidad de los servicios. Por otra parte, se persigue una mayor adecuación de los servicios a las necesidades del conjunto de la sociedad. Para lograr un aumento en los niveles de eficacia deben tenerse en cuenta los deseos y los objetivos de los consumidores (tanto alumnos como padres), así como proporcionar a los consumidores un abanico de posibilidades de elección más amplio y una mayor dependencia de los proveedores (centros) con respecto a los consumidores (estudiantes).

Con respecto a la equidad, a través de los mecanismos de cuasimercado debe buscarse un equilibrio que permita mantener el mismo nivel de igualdad de posibilidades de acceso a los servicios educativos. Bajo este criterio, el sistema público (para la enseñanza obligatoria) proporcionaría un currículum común, gratuidad de la enseñanza, acceso al mismo tipo de instituciones, etc.

Como en la mayor parte de las decisiones públicas en el terreno de los cuasimercados surgen de nuevo conflictos entre los criterios de eficiencia y equidad. En el caso de la educación el conflicto se plantea entre capacidad de elección y equidad entendida como igualdad de acceso. Siguiendo a Calero (1997), los elementos teóricos de un sistema de cuasimercado relacionados con los incentivos a la eficiencia y a la equidad pueden resumirse en los siguientes aspectos:

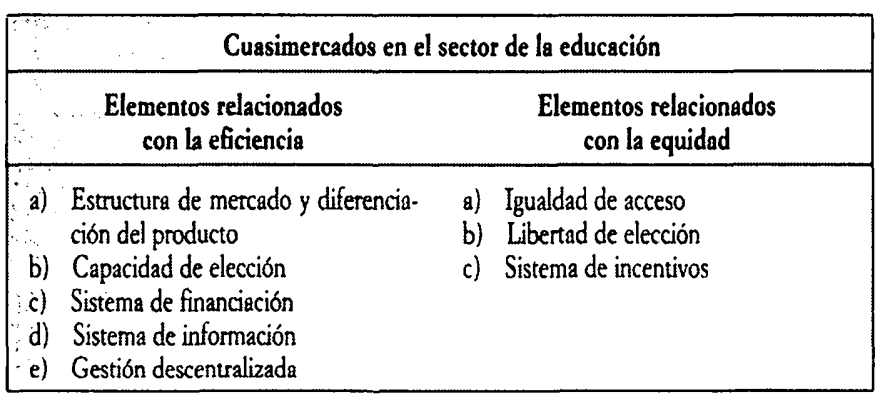

a) Estructura de mercado y diferenciación del producto: para que un cuasimercado funcione de forma adecuada es necesaria la presencia de diversos centros escolares que puedan ofrecer un producto diferenciado y que compitan entre sí para conseguir las matrículas de los alumnos. En el mercado educativo, generalmente aparece una estructura de mercado con poca concentración de oferentes y demandantes, excepto en el caso de los niveles superiores de enseñanza. Además, la diferenciación de productos está limitada por la existencia de curricula únicos. A pesar de esto, pueden establecerse elementos diferenciadores que permitan la competencia entre los centros ofreciendo productos distintos, a través de numerosos instrumentos, como las actividades extraescolares, el profesorado con que cuenta el centro, las instalaciones, etc. 
b) Capacidad de elección del centro por parte del usuario, o «matrícula abierta», por parte del proveedor: La posibilidad de matricularse en cualquier centro, independientemente de la distancia que exista en relación al lugar de residencia del alumno, favorece la eficiencia del sistema al fomentar la competencia entre los centros escolares y potenciar a los «mejores» centros en términos de calidad y de costes. Sin embargo, en un ámbito como el educativo - en el que las variables de contexto desempeñan un papel fundamental en los resultados logrados por las distintas unidades escolares- dicha elección puede deberse a una serie de factores que podrían distorsionar el funcionamiento del mercado, como puede ser la elección del centro en función del alumnado o del «prestigio social» del centro.

c) Sistemas de financiación: el funcionamiento del cuasimercado será mejor cuanto más estrecha sea la relación existente entre el comportamiento de los centros y la financiación pública recibida por los mismos. Para que los centros que exhiben mejores comportamientos puedan recibir un nivel mayor de recursos públicos existen, principalmente, dos vías alternativas: fórmulas que incorporen más recursos cuanto más alumnos sean atraídos por los centros o fórmulas basadas en vales educativos.

d) Sistemas de información y evaluación de la calidad: la información sobre las características de los centros en un entorno de cuasimercado tiene una doble vertiente: la publicidad propia de los centros para atraer a los alumnos y las evaluaciones homogéneas controladas por un regulador que distribuya tales informaciones. La combinación de estos dos aspectos resulta de crucial importancia para que los alumnos —o sus familias - puedan realizar lä elección del centro que prefieren.

e) Gestión descentralizada: se consigue diferenciar el producto y hacer responsable a cada centro de los beneficios y pérdidas generados por el proceso de producción. La emulación del mercado en este caso requiere que los proveedores del servicio educativo reciban incentivos o desincentivos en función de su capacidad de atraer usuarios. La posibilidad de «salida» de los usuarios implica que los centros poco eficientes deberían interrumpir su actividad. No obstante, como señala GLENNERS. TER (1993), se produce una situación de costes no recuperables en la medida que los costes sociales generados por el cierre de una escuela pueden ser muy elevados.

Desde el punto de vista de la equidad, la aplicación de cuasimercados educativos puede conducir a una reducción de la equidad global en tres ámbitos:

a) Igualdad de acceso: la separación entre la financiación y la producción de los servicios públicos no debería generar diferencias en el caso de competencia entre oferentes públicos y privados. Sin embargo, puede ocurrir que determinados grupos sociales vean dificultado su acceso a los centros por el esta- blecimiento de precios de matrícula. Este inconveniente podría ser fácilmente superado estableciendo limitaciones en los precios para aquellos centros que deseen recibir financiación pública (de forma similar a la arbitrada para los centros concertados en nuestro país) o estableciendo mecanismos compensatorios destinados a evitar que los alumnos pertenecientes a familias con menores recursos económicos vean mermada su capacidad de elección de centro.

b) Libertad de elección por parte del oferente: en el caso de que los centros que tengan mayor demanda que capacidad puedan rechazar a aquellos alumnos que por sus características encarezcan el proceso productivo de prestación del servicio, se producirán sesgos que favorecerán a los alumnos «más aventajados y capaces» (hipótesis de selección o cream-skimming). En este sentido, como pone de manifiesto VILLARROYa (1999), el establecimiento de un cuasimercado no produce un resultado neutro en las posibilidades de selección, especialmente en aquellos centros que pueden utilizar métodos informales en la admisión de alumnos. Así, por ejemplo, en el caso del acceso a los centros españoles sostenidos con fondos públicos, los criterios actualmente vigentes para regular la admisión de alumnos podrían estar generando efectos no deseados.

El problema de la selección es, probablemente, el más difícil de solucionar de entre los que pueden originarse como consecuencia de mecanismos de cuasimercado en el ámbito educativo, en la medida que los instrumentos de financiación tienen una incidencia muy limitada en este sentido. Ello es debido al reducido efecto de los centros educativos sobre los resultados académicos de los alumnos. Esta circunstancia clarifica dos aspectos. Por una parte, los incentivos que tienen los administradores de centros para intentar seleccionar a los alumnos de determinados grupos sociales. Por otra, el reducido papel que puede tener la financiación como medio para desincentivar la tendencia a la selección. Por tanto, parece sensato que la solución para el problema de la selección debería pasar más por mecanismos reguladores que establecieran, en condiciones de exceso de demanda, puntuaciones objetivas que permitan establecer prioridades en la lista de acceso y no por mecanismos de financiación compensadora para los centros.

c) Sistema de incentivos: los esquemas de incentivos que se establezcan pueden tener importantes efectos sobre la equidad en la medida que el sistema permita que la financiación pública sea complementada con recursos privados. En este caso, los incentivos monetarios recibidos por el profesorado pueden depender tanto de su calidad docente como del nivel económico de las familias de los alumnos del centro en el que desarrollan su labor docente. 


\section{La experiencia educativa británica: mecanismos de competencia gestionada y mercados internos}

El «mercado educativo» en el Reino Unido tiene algunas características distintivas ${ }^{1}$ que deben ser tenidas en cuenta antes de estudiar, interpretar y evaluar el establecimiento de los mecanismos de cuasimercado desarrollados en el mismo tras la aplicación de la reforma:

1. Los incentivos para la maximización de beneficios o para la minimización de costes son muy débiles y resulta difícil para cualquiera de las partes apropiarse de cualquier excedente que pueda generarse, al menos en términos monetarios.

2. El mercado es competitivo en la medida en que existen muchos centros, pero la expansión, las fusiones y las entradas y salidas del mercado están muy limitadas.

3. Algunos centros educativos pueden tener un monopolio espacial, ya que uno de los mayores costes para los consumidores son los costes de desplazamiento, tanto monetarios como no monetarios.

4. Los centros educativos públicos no pueden competir en precios, pero sí en calidad, medida a través de los resultados reflejados en las tablas de clasificación (league tables).

5. La mayoría de las escuelas tiene muy poca elección sobre la calidad de los inputs, sobre el nivel de output o sobre los precios.

6. Es un mercado fuertemente regulado por el gobierno (tanto central como local) debido tanto a la existencia de efectos externos como a la consecución de determinados objetivos de equidad.

Como señala ADNETT (1998), los argumentos en los que se ha basado la reforma del sistema educativo en el Reino Unido se han basado en tres grupos de ideas claramente provenientes de distintas instituciones. En primer lugar, el Instituto Nacional para la Investigación Económica y Social (National Institute of Economic and Social Research) ha puesto de manifiesto sistemáticamente, en un amplio número de estudios, la escasa capacidad de la economía británica para hacer frente a los fallos del sistema educativo. En segundo lugar, el Instituto de Asuntos Económicos (Institute for Economic Affairs), también en diversos trabajos e informes elaborados a partir de la década de los setenta, ha realizado una fuerte crítica al sistema educativo británico ${ }^{2}$ proponiendo la adopción de mecanismos basados en el mercado. Finalmente, y basándose en la literatura desarro. llada a partir del Informe Coleman (1966), algunos economistas han incidido en la débil relación que existe entre las diferencias en la financiación de los distintos centros educativos y la calidad del output obtenido por los mismos, tal y como señalan numerosos estudios empíricos ${ }^{3}$. Esta débil relación entre los recursos empleados y los resultados obtenidos ha sido interpretada por algunos autores (HANUSHEK, 1996) como un reflejo de la ausencia de incentivos de mercado en el sistema educativo.

\subsection{El contenido de la Reforma británica}

Como consecuencia de estas recomendaciones, a raíz de la Education Reform Act de 1988, prácticamente todos los mecanismos usados para proveer fondos para la educación en el Reino Unido cambiaron drásticamente en todos los niveles, pero particularmente en la educación primaria y secundaria. Además de estas alteraciones a las que nos referiremos a continuación, tuvieron lugar otros cambios en la misma línea, como son la introducción de las tablas de clasificación para los centros de educación secundaria y del currículum común (National Curriculum) para estudiantes entre cinco y dieciséis años.

En lo que a financiación se refiere, ha habido alteraciones en la fórmula de reparto de los fondos para la financiación de la educación correspondiente al nivel local (Local Education Autborities School- LEA Schools). Un sistema paralelo de financiación para los centros con «gobierno autónomo» (Grant Maintained Schools- GM Schools) ha sido implantado con el objeto de trasladar la financiación basada en decisiones del gobierno local acerca del nivel de gastos hacia fórmulas de financiación «comunes». Esta fórmula innovadora de reparto comenzó a aplicarse por primera vez en abril de 1994 en cinco áreas piloto de Inglaterra y posteriormente se extendió hasta 21 entre 1995 y 1996. Se espera que con el tiempo sea reemplazada por una fórmula de financiación «nacional» (National Funding FormulaNFF).

En consecuencia, la Education Reform Act (1988) disminuyó de forma considerable el control administrativo de las autoridades locales sobre los centros educativos. Con esta reforma llevada a cabo por la Administración conservadora, lo que se pretendía era incrementar los niveles de elección de los padres a la hora de elegir la escuela de sus hijos y potenciar la competencia entre las escuelas por conseguir el mayor número posible de alumnos. En concreto, los pilares fundamentales de la reforma fueron los siguientes:

a) Gestión local de las escuelas, bajo la cual los centros educativos pasaron a recibir la mayor parte de su financiación en función del número de alumnos matriculados en el centro y de sus edades, a la vez que se les concede un amplio margen de discrecionalidad sobre el empleo que pueden dar a los recur. sos obtenidos. 
b) Procesos de matriculación más abierta, que implica que no se pueden aplicar restricciones en relación al número de alumnos admitidos en cada uno de los centros, operando como único límite la capacidad física de los mismos y también mayor información a los padres.

c) Sistemas de financiación diferenciados, permitiendo a los centros educativos la posibilidad de escapar del control de las autoridades locales y financiarse por transferencias recibidas inicialmente del Ministerio de Educación, y en la actualidad de la Funding Agency for Schools.

Estos mecanismos han supuesto una reducción del control de las autoridades educativas locales sobre los centros educativos, a la vez que se ha incrementado el control por parte del gobierno central, como consecuencia de la introducción del currículum nacional y de la publicación de las tablas de clasificación. La Education Act de 1988 introdujo un currículum nacional, inexistente hasta entonces, con la finalidad de homogeneizar en cierta medida las enseñanzas impartidas en todo el territorio nacional, así como la evaluación obligatoria de los centros y la publicación de tablas de clasificación de los centros, elaboradas a partir de una serie muy amplia de indicadores de gestión, con la finalidad de ofrecer a los padres una amplia información sobre la cual poder realizar su elección. En este mismo sentido, en el año 1992 se creó un nuevo organismo central de regulación encargado de organizar la inspección de los centros educativos cada cuatro años.

\section{a) Gestión local de las escuelas}

Antes de la introducción de la Gestión local de las escuelas, los recursos recibidos por cada uno de los centros eran asignados por las autoridades locales educativas en función del coste de los inputs, entre los cuales el más importante era la remuneración de los profesores.

El principal objetivo del gobierno al introducir la gestión local fue clarificar a los padres y gestores las bases sobre las cuales se calculaban los recursos de cada centro y dar a los mismos la libertad para tomar decisiones de gasto ajustadas a sus prioridades y la capacidad de retener para ejercicios sucesivos las cantidades no gastadas. En este sentido, la gestión local de las escuelas puede ser considerada, en contraste con el mecanismo que le precedía, un sistema de financiación basado en los outputs. De esta forma, el presupuesto de un centro se determina, principalmente, en función del número de alumnos - ponderados por edades-, aunque también se tienen en cuenta necesidades educativas especiales y algunos factores exógenos que escapan al control inmediato de los centros.

$\mathrm{Al}$ introducirse este procedimiento de financiación, el gobierno central limitó significativamente la discrecionalidad de las autoridades locales para asignar los recursos y discriminar sub- jetivamente entre escuelas. A pesar de esto, y como señala BARROW (1998), algunas autoridades locales intentaron reproducir inicialmente los flujos existentes con el sistema anterior, estableciendo las ponderaciones aplicadas a los alumnos exclusivamente con esta finalidad. En definitiva, los esquemas de gestión local se diseñaron en gran medida para reflejar el statu quo existente con anterioridad, y no sobre criterios objetivos que tuvieran en cuenta los costes de enseñanza de los alumnos en función de sus edades.

\section{b) Procesos de matriculación abiertos}

Una de las características más peculiares del sistema de cuasimercado creado en el Reino Unido es intentar evitar la reticencia de las escuelas a expandirse una vez que han admitido el mayor número de alumnos posible. Es difícil encontrar recursos con los que financiar inversiones en inmovilizado y existe, como consecuencia de ello, resistencia a crear comunidades escolares mayores. Esto hace que la competencia por los alumnos quede como un posible estímulo de mejora sólo para aquellas escuelas que tienen plazas vacantes.

Las críticas fundamentales dirigidas al sistema de competencia para atraer al alumnado se han basado principalmente en la posibilidad de que se den comportamientos oportunistas por parte de los centros, seleccionando a los mejores alumnos. Como indica BARTLETT (1993), el sistema actual genera incentivos para que aquellos centros educativos en los que se pro. duzca una fuerte demanda discriminen en favor de los alumnos más capaces y que sean susceptibles de originar problemas menores. En aquellos casos en los que la actuación de los profesores y los centros se evalúe en términos de los logros absolutos alcanzados por los alumnos y no en relación con el valor añadido generado en el proceso educativo, el proceso de discriminación puede verse reforzado; así, los centros tendrán que utilizar menos recursos por alumno para conseguir unos resultados determinados si seleccionan cuidadosamente a su alumnado, evitando a aquellos niños que por sus características socioeconómicas puedan ocasionar problemas mayores. Esto explica la mayor selección efectuada pór los colegios y los crecientes problemas de exclusión a los que se enfrentan algunos alumnos desde que comenzó la publicación de las tablas de clasificación (EDWARDS y WHITTY, 1990).

\section{c) Sistemas de financiación diferenciados}

Uno de los elementos claves de la reforma, y que ha contribuido a crear también algunas distorsiones en el funcionamiento del sistema, es la posibilidad que se concede a los centros de educación secundaria que lo deseen de escapar del control de la autoridad local y autoadministrarse, financiándose 
mediante transferencias recibidas directamente del gobierno central.

El principal objetivo del gobierno al establecer esta posibilidad era aumentar la elección y la diversidad de los centros. La fórmula de asignación de los recursos que se empleó inicialmente para financiar estos centros era una réplica de la utilizada por los centros que optaban por la gestión local. En este sentido, aproximadamente el $85 \%$ de los recursos que recibian dichos centros se calculaba mediante este procedimiento y además recibían una financiación adicional para poder adquirir una serie de servicios que se suministraban de forma gratuita a los centros dependientes de las autoridades locales. De esta forma, se perseguía que la opción por el status de centro financiado mediante transferencias del gobierno central fuera neutral desde el punto de vista financiero.

Sin embargo, en la práctica, muchas de las escuelas que inicialmente optaron por recibir sus fondos directamente del gobierno central obtuvieron niveles de ingresos mayores a los que habrían obtenido si hubieran estado bajo el control de la autoridad local.

A partir del año 1992, y con la finalidad de conseguir el objetivo de neutralidad y de procurar un marco más estable a largo plazo para financiar a los centros dependientes del gobierno central, se estableció un nuevo sistema conocido como Fórmula de Financiación Común. Este sistema que se aplicó como experiencia piloto en cinco autoridades locales en el año 1992 se ha ido extendiendo de forma progresiva a partir de esa fecha y ha pretendido como objetivo último que no se produzcan grandes diferencias entre los centros educativos que optan por una u otra vía de financiación. Sin embargo, como apunta BARTLETT (1993), pueden existir importantes problemas de eficiencia asignativa, derivados de la financiación diferenciada, si consideramos el sistema educativo en su conjunto. En la actualidad, en el Reino Unido las escuelas de educación secundaria están relativamente bien financiadas en relación con los centros de educación primaria, recibiendo aproximadamente un $50 \%$ más de financiación por alumno matriculado. En este contexto, aunque las autoridades locales desearan corregir este desequilibrio, verían seriamente limitadas sus posibilidades de actuación, ya que ello implicaría menos recursos para las escuelas secundarias que verían aumentar sus incentivos para pasar a depender de las transferencias del gobierno central.

\subsection{Valoración de la Reforma}

El mercado interno creado en la educación británica descansa principalmente en los mecanismos establecidos para asignar los recursos y no en un sistema de precios o de preferencias del consumidor. La mayor complejidad de los mecanismos de financiación ha originado resultados positivos así como algunos efectos no deseados, tanto desde el punto de vista de la eficiencia como de la equidad.

El cuasimercado introducido en el sector educativo británico ha alterado de forma significativa la estructura de incentivos, con efectos contrapuestos sobre la eficiencia. Por un lado, el hecho de que los presupuestos escolares sean fijos y los ahorros puedan ser utilizados en ejercicios posteriores puede haber originado incrementos en los niveles de eficiencia. Prueba de ello es el hecho de que los centros educativos acumularon saldos significativos que al final del ejercicio 1993-1994 ascendían aproximadamente a quinientas mil libras esterlinas. Sin embargo, este incentivo podría verse debilitado si las autoridades locales y el gobierno central usaran los altos niveles alcanzados por dichos saldos como una excusa para reducir la financiación en años posteriores. Las importantes diferencias en los niveles de los saldos acumulados por las escuelas ha protegido hasta el momento a todos los centros de que se produzcan los mencionados recortes en los presupuestos educativos.

Las reformas efectuadas han supuesto también una reducción del control burocrático, que se ha visto reemplazado por el control impuesto por los nuevos mecanismos de financiación, en los que el $80 \%$ de los fondos recibidos dependen del número de alumnos matriculados en el centro educativo. Este hecho, unido a los procesos de matriculación abiertos y a la mayor información suministrada a los padres, ha originado fuertes incentivos para que las escuelas incrementen la calidad de los servicios que prestan y realicen actividades encaminadas a atraer el mayor número posible de estudiantes. Sin embargo, hay que señalar que los niveles de competencia a los que se enfrentan cada una de las escuelas están muy condicionados por determinados factores locales, como el número de niños en edad escolar que residen en el área en el que se encuentra situada la misma o como la presencia en las cercanías de otros centros con plazas vacantes.

Como señala GLENNESTER (1991), el nuevo sistema, a pesar de incorporar la competencia entre los centros educativos como una de sus características fundamentales, se encuentra lejos de ser una solución de mercado, ya que, en primer lugar, los fondos públicos destinados a financiar a los centros públicos no pueden ser destinados a centros privados. Además, en segundo lugar, no hay libertad de entrada para nuevos proveedores, en la medi$\mathrm{da}$ en que las inversiones de capital para ampliar los centros existentes o para crear nuevos centros han de ser aprobadas por las autoridades locales y por el Ministerio de Educación. En este sentido, la capacidad de crecimiento de los mejores centros para afrontar una demanda creciente por parte del alumnado está muy restringida. En tercer lugar, las posibilidades de elección de los padres se encuentran también limitadas por el hecho de que, a partir de la reforma, se estableciera un currículum común. Finalmente, los sueldos de los enseñantes -la partida más importante de los centros educativos- se deter- 
mina por una escala fijada a nivel nacional, limitando fuertemente la capacidad de los centros para asignar los recursos de los que disponen.

\section{Consideraciones finales}

El presente trabajo ha pretendido ofrecer una panorámica acerca de las ventajas y los problemas relativos a la implantación de mecanismos de simulación de mercado en el sistema educativo británico. Como indican ADNeTT y Davies (1998), para que las fuerzas de mercado $\multimap$ de cuasimercado - promuevan un resultado eficiente desde el punto de vista social deberían satisfacerse tres conjuntos de condiciones, que en el sector educativo resulta altamente improbable que se cumpla.

En primer lugar, los consumidores necesitarían tener una amplia capacidad de elección entre un conjunto de centros educativos que ofrecieran servicios relativamente diferenciados, así como información adecuada para poder evaluar las diferentes alternativas que se les presentan. Esta información, dada la naturaleza del sector educativo, debería reflejar no sólo los resultados inmediatos obtenidos por los centros, sino también los resultados en el medio y largo plazo.

En segundo lugar, los consumidores deberían poder comunicar sus preferencias a los centros educativos y éstos deberían ser capaces de adaptarse a dichas preferencias. En el cuasimercado educativo, como hemos visto en el presente trabajo, una agencia central (autoridad local o gobierno central) suministra los recursos con los que se financian los centros, imponiendo una serie de restricciones sobre la variedad y la calidad de los productos ofrecidos, sobre la capacidad de los centros para crecer o para reducir su tamaño y sobre los mecanismos de toma de decisiones en el ámbito interno de los colegios. Este alto grado de regulación trata de evitar los problemas que surgen como consecuencia de las fuertes asimetrías de infor- mación propias del sector educativo, pero condiciona notablemente la capacidad de los centros para adaptarse a las preferencias de los consumidores.

Finalmente, la tercera condición necesaria para que los cuasimercados condujeran a un resultado eficiente desde el punto de vista social sería que las preferencias de los consumidores fueran consistentes con la maximización del bienestar social a lo largo del tiempo o que el régimen de regulación establecido ajustara el comportamiento del mercado con la finalidad de lograr dicha consistencia.

En definitiva, dadas las características del sector, no parece que la introducción de los mecanismos de cuasimercado en la prestación de los servicios educativos pueda conducir per se a mejoras en la eficiencia y en la productividad de los recursos empleados. La necesidad de un marco regulador fuerte que limite la libertad de actuación de los proveedores puede hacer que la provisión directa a través del sistema burocrático tradicional conduzca a una asignación de recursos más eficiente que la que se logra introduciendo algunos mecanismos de simulación de mercado. En cualquier caso, el análisis teórico no permite, a priori, determinar los efectos de las reforma sobre la eficiencia de los centros educativos. Sería por ello necesario la realización de estudios de carácter empírico que permitieran evaluar la bondad de las reformas. emprendidas. Resulta sorprendente, en este sentido, que, a pesar del gran número de trabajos teóricos desarrollados sobre los cuasimercados, los estudios empíricos realizados hasta la fecha sobre los efectos de los mismos son muy escasos y se han centrado exclusivamente en la introducción de los mecanismos de cuasimercado en el sistema sanitario. Creemos que, a pesar de las dificultades especificas que conllevan este tipo de análisis en el sector educativo - problemas de selección de las variables, desconocimiento de la función de producción, fuerte influencia de las variables de entorno sobre los resultados obtenidos, etc.-, los resultados de los mismos podrían iluminar el debate actual sobre la conveniencia o no de la utilización de los mecanismos de cuasimercado en el sector de la educación.

\footnotetext{
- Departamento de Economía Aplicada, Universidad de Extremadura.

- Departamento de Economía y Hacienda Pública, Universidad Autónoma de Madrid.

' Vére Barrow (1998).

2 Con anterioridad a la reforma, la escuela pública funcionaba como un monopolio local, en el cual los alumnos se asignaban a las escuelas en función de
}

su lugar de residencia. Como algunos autores señalan [ver, por ejemplo, СнuBB y MOE (1988) o FELS (1995)], esta estructura institucional originaba tanto ineficiencia asignativa como ineficiencia de tipo $\mathrm{X}$, debido a la inexistencia de incentivos para que funcionarios y profesores respondieran a las demandas sociales.

${ }^{3}$ Ver, por ejemplo, Hanushek $(1986,1996)$ o Haveman y Wolfe (1995). 
Albi, E.; González-Páramo, J. M., y López I Casasnovas, G. (1997), Gestión Pública. Fundamentos, Técnicas y Casos, Ed. Ariel.

AdNETT, N. (1998): «Competition in the School Curriculum: the Economic and Policy Context in the UK», Working Paper, Staffordshire University Business School.

Adnett, N., y DaVtes, P. (1998), "Reconciling the economic and sociological analyses of UK schooling quasi-markets», Working Paper, Staffordshire University Business School.

BARTLETT, W. (1993), Quasi-Markets and Educational Reforms, en LE GRAND y W. BarTleTt (eds.), Quasi-Markets and Social Policy (London: Macmillan), cap. 6.

Barthett, W.; RoberTs, J. A., y Le Grand, J. (1998), Quasi Markets Reforms in the 1990s. A Revolution in Social Policy, University of Bristol: The Policy Press.

CALERO, J. (1998), Una evaluación de los cuasimercados como instrumento para la reforma del sector público, Fundación BBV.

ChUBB, J., y MoE, T. (1988), «Politics, Markets and the organization of Schools», American Political Science Review, vol. 82, No. 4, pp. 1065-1087.

Cums, J. G., y Jones, P. R. (1997), «Los Cuasimercados desde la Perspectiva de la Elección Pública», Hacienda Pública Española, núm. 140, pp. 39.51.

GLENNERSTER, H. (1991), «Quasi-markets for education?», Economic Joumal, 101, pp. 1268.1276.

EDwARDS, T., y WHITTY, D. (1997), «Marketing Quality: Traditional and Modern Versions of Educational Excellence», en Glatter, Woods y Bagley (eds.), Choice and Diversity in Schooling: Perspectives and Prospects, Routledge.

FELS, R. (1995), «Making US schools competitive», en BECKER y BAUMOL (eds.), Assessing Educational Practices: The Contribution of Economics, MIT Press, Russell Sage Foundation.
Hanushex, E. (1986), «The Economics of Schooling: Production and Efficiency in Public Schools», Joumal of Economic Literature, vol. 24, pp. 1141-1177.

HANUSHEK, E. (1996), «Measuring Investment in Education», Joumal of Economic Perspectives, vol. 10, No. 4, pp. 9.30.

HaVeman, R, y Wolfe, B. (1995), «The determinants of children's attainments: a review of methods and findings", Joumal of Economic Literature, vol. 33, pp. 1829-1878.

Hughes, D.; GruFfTTH, L., y MCHALE, J. V. (1997), «Do Quasi-markets Evolve? Institutional Analysis and the NHS», Cambridge Joumal of Economics, 21, pp. 259.276

JÉRÒME-ForGeT, M.; WHTtE, J., y WIENER, J. M. (eds.) (1996), Intemal Markets Experience and Proposals, Washington, DC: The Brooking Institution.

Levacic, R. (1994), «Evaluating the Performance of Quasi-markets in Education», en Bartett, Propper, Wilson y Le Grand (eds.), Quasi-markets in the Welfare State, SAUS Publications.

POWER, S.; HALPIN, D., y WHITTY, G. (1997), «Managing the State and the Market: "New" Education Management in Five Countries», British Joumal of Educational Studies, vol. 45, No. 4, pp. 342.62.

Villaroya, A. (1999), «Algunas reflexiones sobre los problemas de selección en el sector de la enseñanza privada concertada en Españan, Actas de las VIII Jormadas de la Asociación de la Economia de la Educación, Ed. Universidad de Alcalá.

WALFORD, G. (1996), «School Choice and the Quasi-market in England and Wales», Oxford Studies in Comparative Education, vol. 6, No. 1, pp. 49-62.

West, A., y Pennell, H. (1997), «Educational Reform and School Choice in England and Wales», Education Economics, vol. 5, No. 3, pp. 285.305. 